\title{
The Novel Bilateral Quadratic Interpolation Image Super-resolution Algorithm
}

\author{
Gengyi Liu \\ School of Information Science and Engineering, Southeast University, Nanjing, 211189, China \\ Email:213183289@seu.edu.cn
}

Received: 17 October 2020; Accepted: 13 November 2020; Published: 08 June 2021

\begin{abstract}
As a method of processing images, image interpolation has been widely applied to image processing. This paper proposed a new method of image super-resolution algorithm based on bilateral quadratic interpolation. We translate interpolation areas of the pixels to the specified area to construct the bilateral quadratic interpolation surfaces. The constructed surfaces are used to estimate the pixel values of the compensating pixel areas. By replacing each pixel with the corresponding areas, the image is amplified. The amplified images of the algorithm have more details remained than the results of the common algorithms. And this novel algorithm has a better improvement in the fidelity of the images. Moreover, it has a better performance in running speed and the quality of the images such as PSNR and SSIM. It can be used on the amplification of the color images, which can provide better quality amplified images for people. And it makes it convenient for people to study carefully on partial information of images.
\end{abstract}

Index Terms: Image processing, quadratic interpolation, translate pixel, image interpolation, image super-resolution algorithm.

\section{Introduction}

As an important part of image processing, image amplification has been widely used in scientific research such as media entertainment [1,2], satellite imaging [3,4], etc. High-resolution images contain more information and have better visual effects. Image super-resolution can resize the images to fit different needs when it comes to complex image processing. Image interpolation is a common method of image super-resolution. Based on different needs, different image interpolation methods will result in different amplified images. These methods can differ from computing time and these images can differ from their features. Therefore, image interpolation plays an important role in image processing, which cannot be underestimated in the field of 4K TV [5], image resolution conversion [6], high-resolution scene reconstruction of games [7], etc.

At present, image super-resolution can be divided into several categories depending on different inputs and different outputs. Input a series of low-resolution images (a low-resolution video) and get a high-resolution image, which is called Reconstruction-based super-resolution. When both input and output are videos, it is called video superresolution. When both input and output are single images, it is called single image super-resolution. Depending on whether to use learning samples, super-resolution can be divided into edge-focused super-resolution and learning-based super-resolution.

Many different methods have been put forward and each of them has its advantages and disadvantages. To get high-quality images, a good and suitable image interpolation method is needed. To preserve texture details of the image, Song et al use contours to divide the image into texture area and smooth area [8]. Each area uses different interpolation models to construct its amplifying area [9]. Thus, a multi-scale detail enhanced image is constructed. Zhou et al make use of 3D modeling theory to amplify the bitmap images. Combine the 3D model of the discrete algorithm with the scanning line compensation algorithm and segment the bitmap to construct the needed model. And finally, get a relatively clear amplified bitmap image [10]. Karim employs an algorithm that constructs a rational bi-quartic spline interpolation scheme. The rational bi-quartic spline has six free parameters and it is used to improve the final interpolation surface. With the final surface, the scheme has higher PSNR and gets better quality enlarged images [11]. Jiang et al make packet loss compensation an alternative method to the traditional reconstruction process. They define a pixel span function to obtain the missing image details. By using this interpolation method, the output images achieve both higher quantitative and qualitative performance results and provide better visual effects [12].

Universally, PSNR (Peak Signal to Noise Ratio) and SSIM (Structural Similarity Index Measure) are used to evaluate the accuracy of image super-resolution algorithm, and the running time to evaluate the complexity of the algorithm [13,14]. The common algorithms are as follows: Bicubic, ANR, NE+LLE, A+ [15-18], etc. Bicubic is the 
traditional interpolation algorithm. ANR, NE+LLE, and A+ depend on machine learning to improve the resolution of the images. However, these algorithms don't work very well when it comes to PSNR, SSIM, and running speed. This paper mainly focuses on single-image super-resolution based on image interpolation. We want to use a novel method to amplify the images with higher PSNR, SSIM, and lower running time than the common algorithms. This paper proposes a novel method of image super-resolution algorithm based on bilateral quadratic interpolation.

\section{Algorithm and Implementation}

\section{A. Basic process of the Algorithm}

The flow chart of the basic algorithm is shown in Fig. 1. First, we extract the pixel values of the low-resolution image. Then, for image edges, construct the interpolation area by adding pixels beside the edges. We can translate the corresponding interpolation area of every pixel to the fixed area to construct the bilateral quadratic surface, which can be used to estimate the compensating pixel values. After that, we combine the compensating pixel values to construct the compensating pixel blocks. Finally, replace the former pixel value with the compensating pixel block. Thus, a highresolution amplified image is constructed.

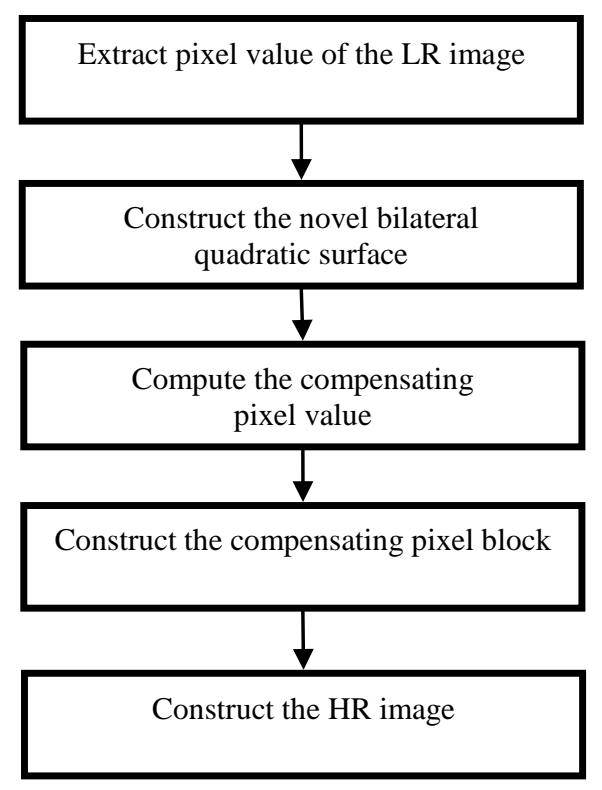

Fig. 1. Algorithm flow chart

\section{B. Construction of the Novel Bilateral Quadratic Surface}

For the LR image, the added edges are added around the image, and the image including the added edge is used to construct the bilateral quadratic surface. Fig. 2 shows the LR image (Fig. 2(a)) and the image including the added edge for the LR image with the size $3 * 4$. Extract the pixel value of each pixel point $(i, j)$ of the image to be enlarged. For the edge of the image, add a pixel-made rectangular border to the image. The pixel values of the border are taken from adjacent pixel values.

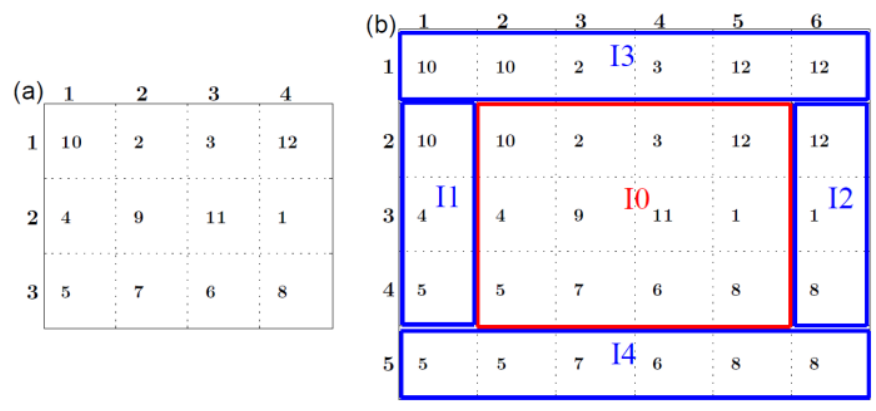

Fig. 2. Image edge process 
For Fig. 2(b), I0 represents the original LR image, I1, I2, I3, I4 are the added edge pixel. In this way, every pixel of the original LR image can have its corresponding interpolation area. $(i, j)$ represents the pixel point which is going to be amplified. Fig. 3 is the interpolation area and translation interpolation area corresponding to the pixel point $(i, j)$.

\begin{tabular}{|c|c|c|}
\hline$(\mathrm{i}-1, \mathrm{j}-1)$ & $(\mathrm{i}-1, \mathrm{j})$ & $(\mathrm{i}-1, \mathrm{j}+1)$ \\
\hline$(\mathrm{i}, \mathrm{j}-1)$ & $(\mathrm{i}, \mathrm{j})$ & $(\mathrm{i}, \mathrm{j}+1)$ \\
\hline$(\mathrm{i}+1, \mathrm{j}-1)$ & $(\mathrm{i}+1, \mathrm{j})$ & $(\mathrm{i}+1, \mathrm{j}+1)$ \\
\hline
\end{tabular}

\begin{tabular}{|l|l|l|}
\hline$(-1,-1)$ & $(-1,0)$ & $(-1,+1)$ \\
\hline$(0,-1)$ & $(0,0)$ & $(0,+1)$ \\
\hline$(+1,-1)$ & $(+1,0)$ & $(+1,+1)$ \\
\hline
\end{tabular}

Fig. 3. The interpolation area and translated Interpolation area

The algorithm will be explained in detail through an example.

Fig. 2 shows the process of image edge processing of a $3 * 4$ image, which adds the row and column of the corresponding image edge pixel. Thus, the image size is changed from $3 * 4$ to $5 * 6$. Next is to extract the pixel value from the interpolation area of the image to be amplified. The process can be represented with Fig. 4, which includes (a) (b) (c) (d) (e) (f) (g) (h) (i) 9 figures. The corresponding coordinates of every figure are the pixel values of the interpolation area corresponding to the pixel point $(i, j)$. For example, the pixel values of the interpolation area corresponding to the pixel $(2,3)$ are distributed to 9 figures and their coordinates in the figures are the same $(2,3)$. According to Fig. 2, we can get the pixel values of the interpolation area of $(2,3)$. They are 2, 3, 12, 9, 11, 1, 7, 6, and 8. These pixel values also correspond to the pixel values of the translate interpolation area.
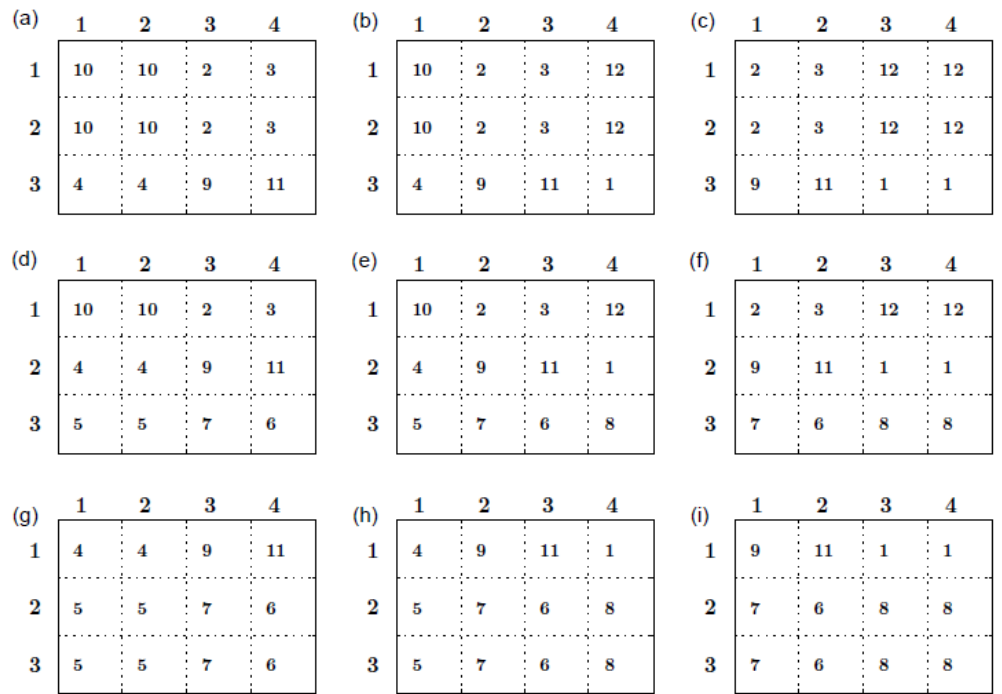

Fig. 4. Extracting process of the pixel value

Fig. 5 shows the matrix made up of the pixel values of corresponding interpolation areas which are extracted from all pixels of the image to be amplified. Every column of the matrix corresponds to the interpolation area of every pixel. The red area is the pixel values of the interpolation area of the pixel point $(2,3)$ in the image to be amplified.

By translating interpolation areas and their pixel values, the quadratic interpolation surface $\mathrm{S}$ is constructed for the interpolation area corresponding to the pixel point $(2,3)$. For the different pixel points, the different quadratic interpolation surfaces are constructed. For the pixel point $(i, j)$, the quadratic interpolation surface $\mathrm{S}$ is constructed by the following steps. 


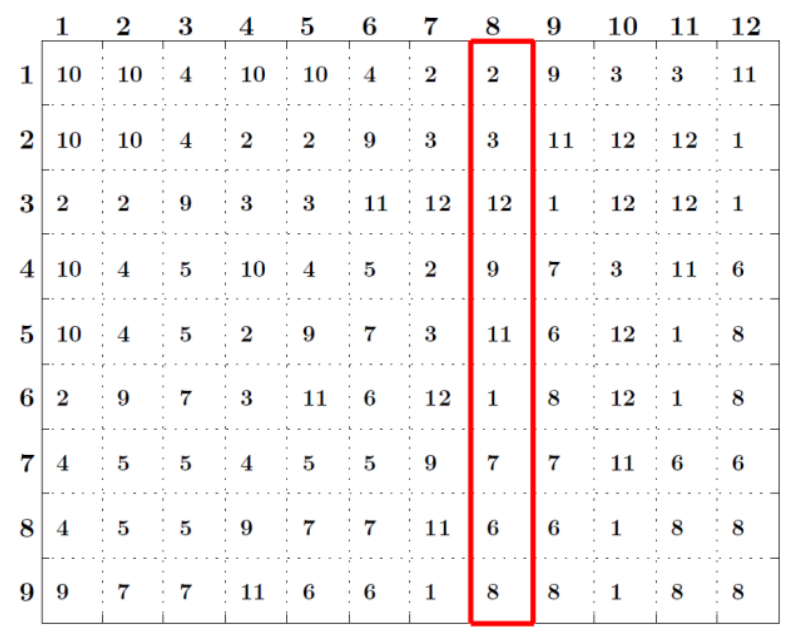

Fig. 5. The matrix composed of the pixel value of the interpolation area

The steps of establishing the model of surface $S$ are as follows:

Step1: Move the interpolation areas of the pixel $(i, j),[i-1, i+1] \times[j-1, j+1]$, to the left by $i$ units and move it up by $j$ units. After the translation, the interpolation area is $[-1,+1] \times[-1,+1]$, and the pixel values remain unchanged.

Step2: Construct bilateral quadratic interpolation surface and its expression is:

$$
\begin{aligned}
& S(x, y)=a_{00}+a_{01} y+a_{02} y^{2}+a_{10} x+a_{11} x y \\
& +a_{12} x y^{2}+a_{20} x^{2}+a_{21} x^{2} y+a_{22} x^{2} y^{2}
\end{aligned}
$$

$S(x, y)$ represents the pixel value of the pixel $(x, y) . x=-1,0,1$, and $y=-1,0,2 . a_{s t}(s=0,1,2, t=0,1,2)$ is undetermined coefficients. For different $x$ and $y$, there are 9 formulas the same as (1), these 9 formulas are represented as the following matric.

$$
T a=S
$$

Where

$$
\begin{aligned}
& T=\left(\begin{array}{lllllllll}
1 & -1 & 1 & -1 & 1 & -1 & 1 & -1 & 1 \\
1 & 0 & 0 & -1 & 0 & 0 & 1 & 0 & 0 \\
1 & 1 & 1 & -1 & -1 & -1 & 1 & 1 & 1 \\
1 & -1 & 1 & 0 & 0 & 0 & 0 & 0 & 0 \\
1 & 0 & 0 & 0 & 0 & 0 & 0 & 0 & 0 \\
1 & 1 & 1 & 0 & 0 & 0 & 0 & 0 & 0 \\
1 & -1 & 1 & 1 & -1 & 1 & 1 & -1 & 1 \\
1 & 0 & 0 & 1 & 0 & 0 & 1 & 0 & 0 \\
1 & 1 & 1 & 1 & 1 & 1 & 1 & 1 & 1
\end{array}\right) \\
& a=\left(\begin{array}{l}
a_{00} \\
a_{01} \\
a_{02} \\
a_{10} \\
a_{11} \\
a_{12} \\
a_{20} \\
a_{21} \\
a_{22}
\end{array}|, \quad S=| \begin{array}{l}
S(-1,-1) \\
S(-1,0) \\
S(-1,1) \\
S(0,-1) \\
S(0,0) \\
S(0,1) \\
S(1,-1) \\
S(1,0) \\
S(1,1)
\end{array} \mid\right.
\end{aligned}
$$

That is, constructing the surface is solving for the values of $a_{s t}$.

Step3: Solve the linear equation (2) to get the value of the undetermined coefficient

$$
a=T^{-1} S
$$




$T^{-1}=\left(\begin{array}{lllllllll}0 & 0 & 0 & 0 & 1 & 0 & 0 & 0 & 0 \\ 0 & 0 & 0 & -0.5 & 0 & 0.5 & 0 & 0 & 0 \\ 0 & 0 & 0 & 0.5 & -1 & 0.5 & 0 & 0 & 0 \\ 0 & -0.5 & 0 & 0 & 0 & 0 & 0 & 0.5 & 0 \\ 0.25 & 0 & -0.25 & 0 & 0 & 0 & -0.25 & 0 & 0.25 \\ -0.25 & 0.5 & -0.25 & 0 & 0 & 0 & 0.25 & -0.5 & 0.25 \\ 0 & 0.5 & 0 & 0 & -1 & 0 & 0 & 0.5 & 0 \\ -0.25 & 0 & 0.25 & 0.5 & 0 & -0.5 & -0.25 & 0 & 0.25 \\ 0.25 & -0.5 & 0.25 & -0.5 & 1 & -0.5 & 0.25 & -0.5 & 0.25\end{array}\right)$

Therefore, the matrix expression of the interpolation surface is:

$$
f(x, y)=T^{-1} S \beta
$$

Where

$$
\beta=\left(1, y, y^{2}, x, x y, x y^{2}, x^{2}, x^{2} y, x^{2} y^{2}\right)^{\prime}
$$

And finally, a surface model is generated.

Then use the novel bilateral quadratic interpolation surface to compute the compensating pixel values of the pixel compensating area $[i, i+1] \times[j, j+1]$.

\section{Compensation by Interpolation Surface}

The same translation strategy as the construction of the interpolation surface, the translated pixel compensating area of the compensating area $[i, i+1] \times[j, j+1]$ is $[0,1] \times[0,1]$. During the pixel compensation, the translated pixel compensating area is divided into the mesh according to the amplifying factor $M$. Namely, $x=(s-1) / M, y=(t-1) / M$, where $s=1, \ldots, M, t=1, \ldots, M$. For the selected pixel point $(i, j)$, the pixel values of the interpolation area are converted into a vector $S$, and

$$
\beta=\left(1, y, y^{2}, x, x y, x y^{2}, x^{2}, x^{2} y, x^{2} y^{2}\right)^{\prime}
$$

So, the compensating pixel value of the compensating pixel point $(x, y)$ is estimated by constructed interpolation surface (4).

\section{Experiments}

Compared with the image super-resolution method based on learning and the image super-resolution method based on interpolation, the novel bilateral quadratic interpolation image super-resolution algorithm is proved by the superiority and feasibility.

The machine learning algorithms, such as ANR, NE+LLE, A+, are used to compare with the proposed algorithm, and the traditional interpolation algorithm Bicubic is used to compare with the algorithm. The image set named set14 is used to test the image super-resolution algorithms mentioned above. The PSNR (Peak Signal to Noise Ratio), SSIM (Structural Similarity Index Measure), RT (running time), are used to measure the superiority of the algorithms, including the quantitative indicators for the selected image set, such as PSNR and SSIM, the complexity indicator such as running time. The PSNR and SSIM of the proposed novel bilateral quadratic interpolation are supposed to be higher than the four algorithms mentioned above and its RT is supposed to be lower. The selected algorithms are performed on the image set set14 which is a classic image set for testing image super-resolution algorithm.

From the table 1, we can see that the proposed novel bilateral quadratic interpolation super-resolution algorithm is superior to the other listed algorithms, including the interpolation-based algorithm Bicubic and the learning-based algorithms, such as ANR, NE+LLE, and A+, from the aspect of minimal indicators, mean indicators, and maximal indicators. For example, the minimal PSNR, the mean PSNR, and the maximal PSNR of the proposed novel bilateral quadratic interpolation algorithm are $21.1684,27.3869,31.7136$ which are better than those of the other selected algorithms, such as the minimal PSNR(20.4687), the mean PSNR(25.5513), the maximal PSNR(29.6089) of the Bicubic interpolation algorithm, the minimal PSNR(19.9258), the mean PSNR(25.2016), the maximal PSNR (29.1175) of the learning algorithm ANR. For the other two measure indicators, such as SSIM and RT, the same conclusion as the PSNR indicator is concluded. From the aspects, PSNR, SSIM, and RT, the proposed novel bilateral quadratic interpolation image super-resolution algorithm is better than the other algorithms Bicubic, ANR, NE+LLE, and A+.

Figure 6 shows the visual effects of several image super-resolution algorithms in local areas, PSNR/SSIM is shown in the Figure. In terms of local details, the proposed image super-resolution algorithm is the best one compared with the other algorithms. 
Table 1. Comparison of image super-resolution algorithms for the image set set14.

\begin{tabular}{|c|c|c|c|c|c|c|c|c|c|c|}
\hline \multirow[t]{2}{*}{ SF } & \multirow[t]{2}{*}{ Algorithm } & \multicolumn{3}{|c|}{ PSNR } & \multicolumn{3}{|c|}{ SSIM } & \multicolumn{3}{|c|}{ RT } \\
\hline & & Min & mean & $\max$ & Min & mean & $\max$ & Min & mean & $\max$ \\
\hline \multirow[t]{5}{*}{$\mathrm{M}=2$} & Proposed & 21.1684 & 27.3869 & 31.7136 & 0.6109 & 0.8296 & 0.9466 & 0 & 0.1200 & 0.2652 \\
\hline & Bicubic & 20.4687 & 25.5513 & 29.6089 & 0.5504 & 0.7851 & 0.9254 & 0 & 0.0336 & 0.0624 \\
\hline & ANR & 19.9258 & 25.2016 & 29.1175 & 0.5363 & 0.7783 & 0.9180 & 2.2776 & 5.0868 & 9.0013 \\
\hline & NE+LLE & 20.5024 & 25.7450 & 29.6134 & 0.5502 & 0.7891 & 0.9235 & 3.1044 & 8.0233 & 14.6329 \\
\hline & $\mathrm{A}+$ & 19.8115 & 25.0673 & 28.9463 & 0.5328 & 0.7760 & 0.9166 & 1.9968 & 5.2416 & 8.9389 \\
\hline \multirow[t]{5}{*}{$\mathrm{M}=3$} & Proposed & 19.3229 & 24.5996 & 28.7295 & 0.4486 & 0.7228 & 0.8965 & 0 & 0.0732 & 0.1404 \\
\hline & Bicubic & 18.1872 & 22.4234 & 26.4935 & 0.3704 & 0.6485 & 0.8501 & 0 & 0.0372 & 0.0780 \\
\hline & ANR & 17.8049 & 21.8756 & 25.6851 & 0.3580 & 0.6320 & 0.8391 & 1.0452 & 2.8356 & 4.5084 \\
\hline & NE+LLE & 18.1861 & 22.4162 & 26.4731 & 0.3701 & 0.6477 & 0.8491 & 1.5132 & 4.0320 & 7.5504 \\
\hline & $A+$ & 17.6958 & 21.7023 & 25.4733 & 0.3544 & 0.6290 & 0.8381 & 1.1544 & 2.8932 & 4.9140 \\
\hline \multirow[t]{5}{*}{$\mathrm{M}=4$} & Proposed & 18.5440 & 23.0158 & 27.1566 & 0.3648 & 0.6493 & 0.8529 & 0 & 0.0792 & 0.2808 \\
\hline & Bicubic & 16.5061 & 20.8122 & 25.1307 & 0.2898 & 0.5669 & 0.7901 & 0 & 0.0288 & 0.0936 \\
\hline & ANR & 16.0788 & 20.2499 & 24.3406 & 0.2768 & 0.5488 & 0.7764 & 0.8736 & 2.0148 & 3.7440 \\
\hline & NE+LLE & 16.5054 & 20.8087 & 25.1211 & 0.2897 & 0.5664 & 0.7894 & 0.9360 & 2.6688 & 4.7112 \\
\hline & $\mathrm{A}+$ & 15.9781 & 20.1010 & 24.1491 & 0.2741 & 0.5472 & 0.7758 & 0.7020 & 2.0304 & 3.6036 \\
\hline
\end{tabular}
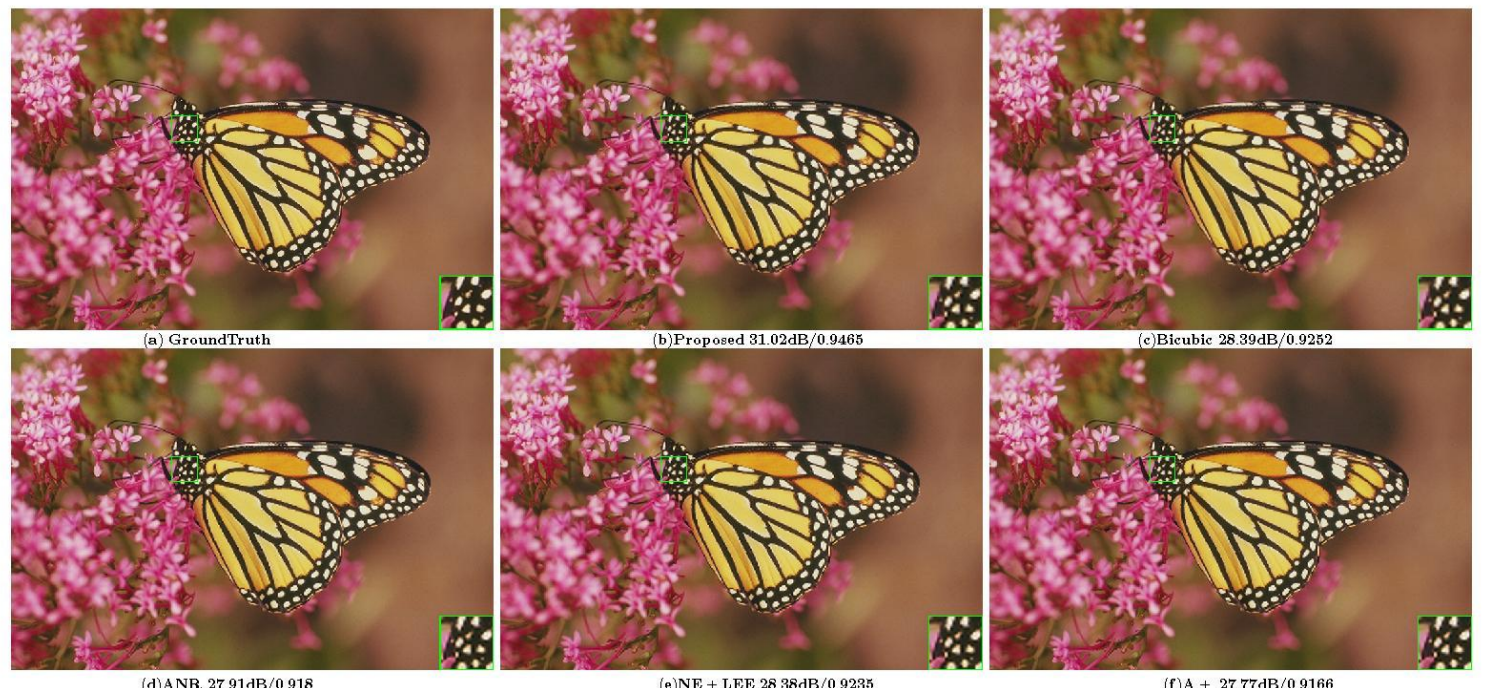

Fig. 6. The visual effects of several image super-resolution algorithms

\section{Conclusion}

In the paper, we presented a novel bilateral quadratic interpolation image super-resolution algorithm, which includes two stages, the first one is the construction of quadratic interpolation surface for the interpolation area corresponding to the pixel of LR image, the second one is the estimation of the pixel values of the compensating area. The proposed algorithm can be applied to the new media, games, and other fields related to image processing. The novel method, translating the interpolation area to the fixed area can be applied to other algorithms, which may make the other algorithms gain better performance in feasibility and efficiency. The experimental results show that the proposed algorithm is better than the other image super-resolution algorithms, such as Bicubic, ANR, NE_LLE, and A+. The proposed algorithm has better performance in PSNR, SSIM, and RT. For future work, the superiority of the proposed algorithm is only proved by the experiment on the selected testing image set set14, this superiority needs to be further verified in reality. More researches are needed to check the superiority of this proposed novel algorithm.

\section{References}

[1] H. Oh, S. Lee, "Visual presence: viewing geometry visual information of UHD S3D entertainment," IEEE Transactions on Image Processing, 2016, 25(7): 3358-3371.

[2] S. Invitto, C. Faggiano, S. Sammarco, V. D. Luca, and L. T. D. P. Haptic, "Virtual interaction and motor imagery: entertainment tools and psychophysiological testing," Sensors, 2016, 16(3): 394. 
[3] Y. Song, Z. Zhou, Z. Zhang, F. Yao, Y. Chen, “A framework involving MEC: imaging satellites mission planning,” Neural Computing and Applications, 2020, 32(19): 15329-15340.

[4] F. Sun, M. Min, D. Qin, F. Wang, J. Hu, "Refined typhoon geometric center derived from a high spatiotemporal resolution geostationary satellite imaging system," IEEE Geoscience and Remote Sensing Letters, 2019, 16(4): 499-503.

[5] W. Weng, J. Lee, C. Dung, "Rapid implementation of 2-dimensional image coding in grand alliance digital television systems," Journal of Information Science and Engineering, 2014, 30(2): 535-546.

[6] Y. Chen, K. Niu, Z. Zeng, Y. Pan, "A wavelet based deep learning method for underwater image super-resolution reconstruction,” IEEE Access, 2020, 8: 117759-117769.

[7] O. Beyersdorff, L. Chew, K. Sreenivasaiah, "A game characterisation of tree-like Q-Resolution size,” Journal of Computer and System Sciences, 2019, 104: 82-101.

[8] G. Song, C. Xiao, X. Li, J. Li, H. Sui, "Structure-preserving texture filtering for adaptive image smoothing," Journal of Visual Languages and Computing, 2018, 45: 17-23.

[9] G. Song, C. Qin, K. Zhang, X. Yao, F. Bao, Y. Zhang, “Adaptive interpolation scheme for image magnification based on local fractal analysis," IEEE Access, 2018, 8: 34326-34338.

[10] L. Zhou, J. Dang, Y. Wang, "Super-resolution amplification of bitmap images based on 3D modeling theory," Journal of Measurement Science and Instrumentation, 2019, 4: 335-341.

[11] S. A. A. Karim, "Rational bi-quartic spline with six parameters for surface interpolation with application in image enlargement," IEEE Access, 2020, 8: 115621 - 115633.

[12] C. Jiang, H. Li, S. Zhou, "Image interpolation model based on packet losing network," Multimedia Tools and Applications, 2020, 79: 25785-25800

[13] Z. Wang, A. C. Bovik, H. R. Sheikh, E. P. Simoncelli, "Image quality assessment: from error visibility to structural similarity," IEEE Transactions on Image Processing,2004, 13(4):600-612.

[14] C. Li, A. C. Bovik, "Content-partitioned structural similarity index for image quality assessment," Signal Processing: Image Communication, 2010, 25(7): 517-526.

[15] R. Timofte, V. D. Smet, L. J. V. Gool, “A+: adjusted anchored neighborhood regression for fast super-resolution,” ACCV 2014, pp. 111-126.

[16] M. Bevilacqua, A. Roumy, C. Guillemot, and M. L. Alberi-Morel, "Low-complexity single-image super-resolution based on nonnegative neighbor embedding,” BMVC2012, Guildford British, pp.1-10.

[17] H. Chang, D. Y. Yeung, and Y. Xiong, "Super-resolution through neighbor embedding," CVPR2004, pp. 275-282.

[18] Q. Zhang, Q. Dou, Y. Yan, W. Hu, "High capacity reversible data hiding algorithm for color image based on bicubic interpolation extension,” Journal of Information Hiding and Multimedia Signal Processing, 2018, 9(1): 61-74.

\section{Author's Profile}

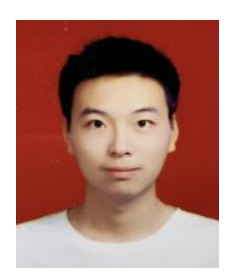

Gengyi Liu, an undergraduate in School of Information Science and Engineering, Southeast University, Nanjing, China. His research interests include digital image processing, image reconstruction and image enhancement.

How to cite this paper: Gengyi Liu, " The Novel Bilateral Quadratic Interpolation Image Super-resolution Algorithm", International Journal of Image, Graphics and Signal Processing(IJIGSP), Vol.13, No.3, pp. 55-61, 2021.DOI: 10.5815/ijigsp.2021.03.05 